\title{
“CRITICAMENTE EM PERIGO" E “AMEAÇADO DE EXTINÇÃO” - STATUS DE ALGUNS ANIMAIS TAXIDERMIZADOS PRESENTES NO MEMORIAL DA EE “CULTO À CIÊNCIA" DE CAMPINAS/SP/BRASIL
}

Tamiris Pinheiro de Souza ${ }^{1}$ PIBIC-EM/CNPq-UNICAMP tamiris.pinheiros@gmail.com

Jovana Cristina Ferreira da Silva ${ }^{2}$ PIBIC-EM/CNPq-UNICAMP jovanacristina039@gmail.com

Cristiana Maria Mendonça Panhan ${ }^{3}$ PIBIC/CNPq-UNICAMP cristianapanhan@gmail.com

Silvia Regina Cason ${ }^{4}$ CIVILIS/FE/UNICAMP silcason@yahoo.com.br

Maria Cristina Menezes ${ }^{5}$

CIVILIS/FE/UNICAMP menezes.mariacristina@gmail.com

O Arquivo Histórico Documental da Escola Estadual "Culto à Ciência" em Campinas/SP/Brasil, local onde se desenvolve o Projeto "Preservação dos Acervos Históricos da EE 'Culto à Ciência'”, coordenado pela Profa. Maria Cristina Menezes, CIVILIS/ FE/UNICAMP, é composto por diversos acervos; dentre os quais está o acervo dos exemplares de animais taxidermizados. Tal acervo desperta estranhamento e curiosidade aos alunos da Instituição e também aos bolsistas do projeto, o que ocasionou a busca por informações sobre este acervo e os espécimes que ele comporta. De início, se pôde constatar por taxidermia.

\footnotetext{
${ }^{1}$ Tamiris Pinheiro de Souza - aluna do Ensino Médio de Campinas - Bolsista no Programa Institucional de Bolsas de Iniciação Científica para o Ensino Médio - PIBIC-EM/CNPq-UNICAMP - CIVILS/FE/UNICAMP. ${ }^{2}$ Jovana Cristina Ferreira da Silva - aluna do ensino médio de Campinas - Bolsista no Programa Institucional de Bolsas de Iniciação Científica para o Ensino Médio - PIBIC-EM/CNPq- UNICAMP - CIVILIS/FE/UNICAMP. ${ }^{3}$ Cristiana Maria Mendonça Panhan - Bolsista Iniciação Científica PIBIC/CNPq - CIVILIS/FE/UNICAMP. ${ }^{4}$ Silvia Regina Cason - doutoranda em Educação - CIVILIS/FE/UNICAMP e Monitora no Programa Institucional de Bolsas de Iniciação Científica para o Ensino Médio - PIBIC-EM/CNPq-UNICAMP.

${ }^{5}$ Maria Cristina Menezes - Professora de História da Educação da FE/UNICAMP e Coordenadora do CIVILIS.
} 
[...] do grego taxi $=$ disposição, e derma $=$ pele), segundo a etimologia, significa disposição da pele. É uma velha arte executada por nossos antepassados, que preservavam tudo quanto admiravam e estimavam ou, pelo menos, o que queriam conservar para a posteridade.

[...] É o caso, por exemplo, de exemplares raros de nossa fauna que, com o avanço cada vez mais acentuado da civilização, tendem a desaparecer. Empregando-se, porém, a taxidermia, (embalsamento) a espécie ficará perpetuada nos museus, escolas e laboratórios.

Portanto, essa arte-ciência, parte integrante de quaisquer culturas, tem por finalidade preservar as espécies raras para estudo e comparações. (Fabichak, 1969,p. 09-10).

Em seguida, se constatou na pasta "IDENTIFICAÇÕES ORIGINAIS DAS ESPÉCIES TAXIDERMIZADAS”, com os espécimes do acervo da Escola, que na mesma figuram dois mamíferos primatas (macaco-prego e bugio) cuja categoria é "ameaçado", e; uma ave aquática (pato mergulhão) cuja categoria é “criticamente em perigo". Então, as atenções se detiveram fortemente sobre os mesmos.

O fato de a categoria, status ou estado de conservação dos animais taxidermizados sofrer variações para determinada espécie conduziu às informações da "NOVA LISTA VERMELHA DE AVES AMEAÇADAS LANÇADA EM DEZEMBRO DE 2016”:

\begin{abstract}
A Lista Vermelha segue critérios precisos para avaliar os riscos de extinção de milhares das espécies no mundo através de categorias criadas que vão de LC (Least Concern - Menor Preocupação), até EX (Extinct - Extinta). Essas categorias permitem informar sobre a urgência para a tomada de medidas de conservação, seja para o público, como para legisladores ou mesmo a comunidade internacional, tentando assim reduzir as extinções.

Nessa nova lista de 2016, 25 espécies brasileiras sofreram alguma alteração de status ou de nome. Dessas, 9 novas espécies entraram para a lista de ameaçadas e 7 saíram da lista ${ }^{6}$.
\end{abstract}

Diante do exposto, seguem descritos os referidos animais taxidermizados do acervo escolar e suas classificações.

\footnotetext{
${ }^{6}$ Nova Lista Vermelha de Aves Ameaçadas Lançada em Dezembro de 2016. Disponível em: $<$ http://www.savebrasil.org.br/listavermelhanewsletter/>. Acesso em: 21 jun. 2017.
} 


\section{MACACO PREGO (nome popular)}

Figura 1. Macaco prego

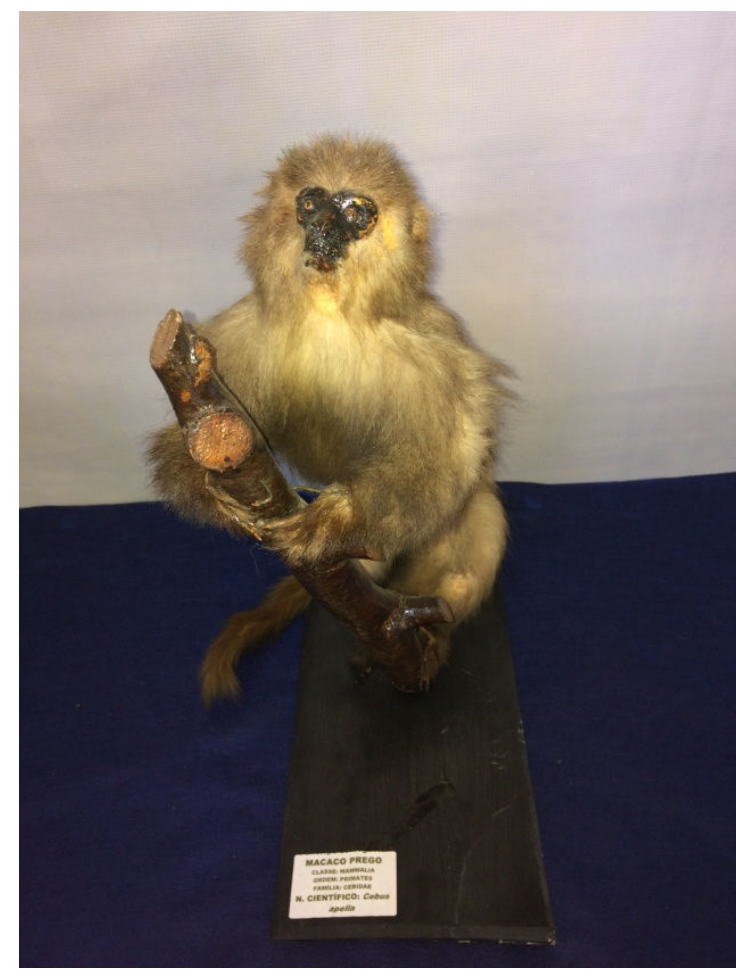

\section{Classificação Cientifica ${ }^{7}$}

Reino: Animalia

Filo: Chordata

Classe: Mammalia

Ordem: Primates

Família: Cebidae

Nome Científico: Cebus apella

Categoria/Estado de Conservação: Ameaçado

Fonte: Arquivo Histórico Documental da EE "Culto à Ciência" Campinas/SP.

Foto: Tamiris Pinheiro de Souza - PIBIC-EM/FAEPEX/UNICAMP.

\section{Conforme a pasta "IDENTIFICAÇÕES ORIGINAIS DAS ESPÉCIES}

TAXIDERMIZADAS", esta espécie encontra-se ameaçada. Ratificando tal dado, a publicação "Mamíferos Ameaçados de Extinção no Brasil" presente no site do Instituto Chico Mendes de Conservação da Biodiversidade (ICMBio), órgão ambiental do governo brasileiro, criado pela lei 11.516 , de 28 de agosto de 2007, afirma que:

[...] Provavelmente abundante no passado, esta espécie de macaco-prego está ameaçada de extinção pelo desmatamento e destruição de seu hábitat (Oliver \& Santos, 1991). Além disso, a intensa pressão de caça e sua captura como animal de estimação, principalmente no Estado da Bahia, vem contribuindo para o declínio das populações. (p.754-757) ${ }^{8}$.

Também foram localizados dados sobre o macaco-prego-dourado ou macaco-pregogalego (nome científico: Sapajus flavius) espécie de macaco-prego, um macaco do Novo Mundo da família Cebidae. Sobre esta espécie Montenegro (2011) diz que:

\footnotetext{
${ }^{7}$ Dados de acordo com pasta "IDENTIFICAÇÕES ORIGINAIS DAS ESPÉCIES TAXIDERMIZADAS". ${ }^{8}$ ICMBIO Instituto Chico Mendes de Conservação da Biodiversidade. Ministério do Meio Ambiente. Disponível em: $\quad<\underline{\text { http://www.icmbio.gov.br/portal/images/stories/biodiversidade/fauna-brasileira/livro- }}$ vermelho/volumeII/Mamiferos.pdf>. Acesso em: 21 jun. 2017.
} 
Apesar de tratar-se de uma espécie recentemente redescoberta, o macaco-pregogalego, Cebus flavius (Schreber, 1774), já figura na lista Vermelha da IUCN como criticamente ameaçado de extinção, principalmente em virtude do reduzido tamanho populacional, da fragmentação e perda de habitat das populações remanescentes e da caça.

O macaco-prego está distribuído geograficamente pelo norte da América do Sul, grande parte do Brasil, até o norte da Argentina.

É um animal muito hábil, que consegue abrir frutas de casca dura. Para essa atividade ele usa pedras e pedaços de pau. São ferramentas rústicas, mas de rara utilização entre animais. Inteligente e de mãos habilidosas, o macaco-prego é facilmente ensinado. Adapta-se ao cativeiro, mas como é muito ativo, frequentemente cria problemas. Nas matas e florestas da América do Sul, vive em bandos [...]. O bando desloca-se continuamente, pulando de galho em galho. A cauda deste macaco não é preênsil. Quando ele se movimenta, mantém a cauda para cima, enrolada como um ponto de interrogação ${ }^{9}$.

\section{PATO MERGULHÃO (nome popular)}

Figura 2. Mergulhão.

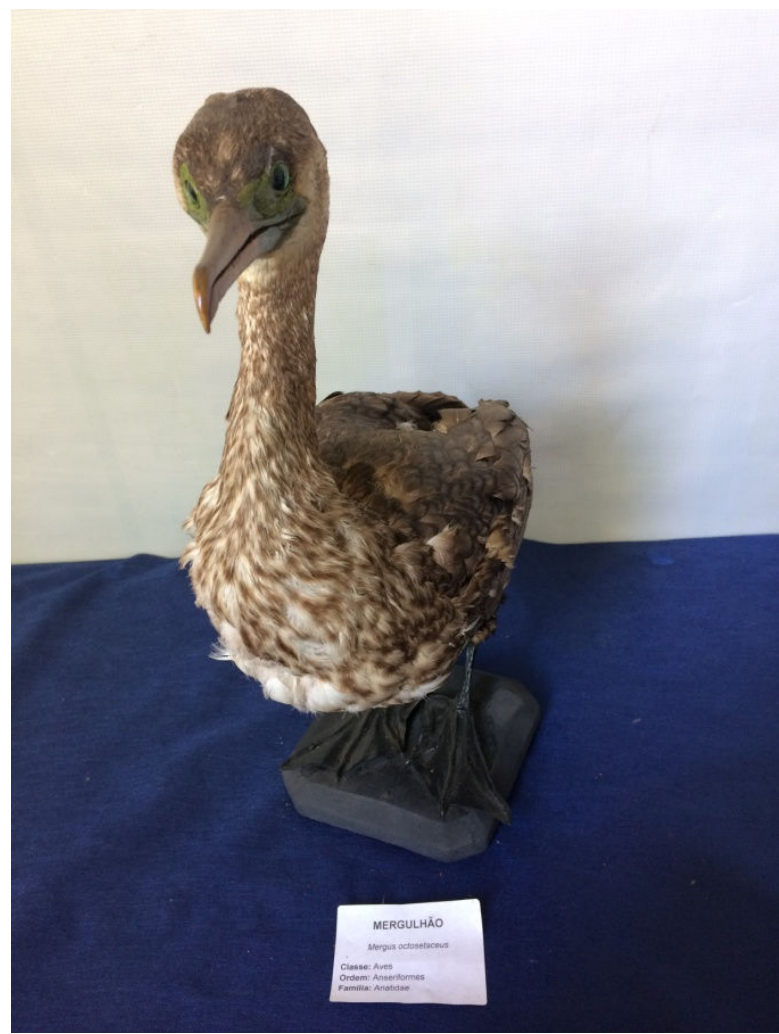

\section{Classificação Cientifica ${ }^{10}$}

Reino: Animalia

Filo: Chordata

Classe: Aves

Ordem: Anseriformes

Família: Anatidae

Nome Científico: Mergus octosetaceus

Categoria: Criticamente em perigo

Fonte: Arquivo Histórico Documental da EE "Culto à Ciência" Campinas/SP.

Foto: Jovana Cristina Ferreira da Silva - PIBIC-EM /FAEPEX/UNICAMP.

\footnotetext{
${ }^{9}$ Tudo sobre o macaco prego. Disponível em: < $\underline{\text { http://temqueserassim.comunidades.net/tudo-sobre-o- }}$ macaco-prego >. Acesso em: 05 jul. 2017.

${ }^{10}$ Dados de acordo com pasta "IDENTIFICAÇÕES ORIGINAIS DAS ESPÉCIES TAXIDERMIZADAS".
}

Rev. Iberoam. Patrim. Histórico-Educativo, Campinas (SP), v. 3, n. 1, p. 238-250, jan./jun. 2017 
Esta ave aquática consta da lista do "Livro Vermelho da Fauna Brasileira Ameaçada de Extinção" ${ }^{11}$ (p.420), conforme mostra a imagem a seguir, sendo classificada como "Ameaçada".

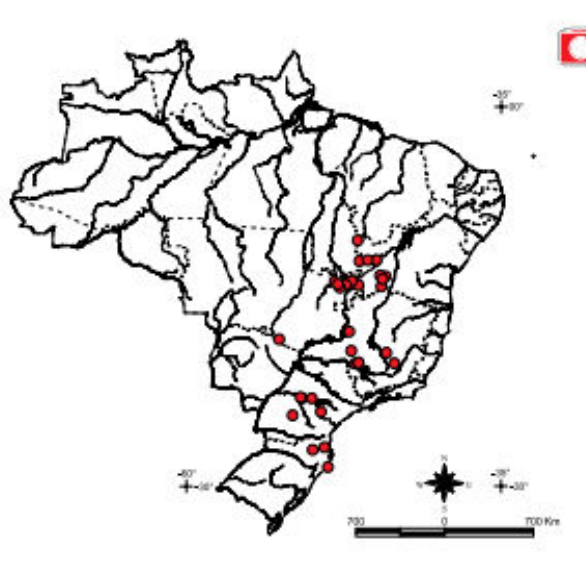

Mergus octosetaceus Vieillot, 1817

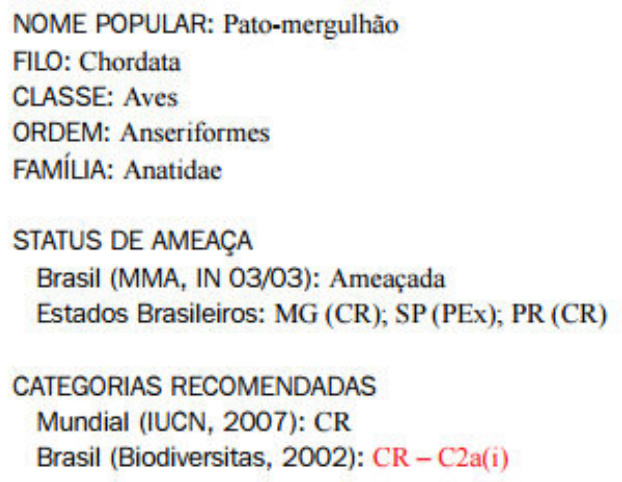

Fonte: Livro Vermelho da Fauna Brasileira Ameaçada de Extinção.

\section{$\mathrm{Na}$ pasta "IDENTIFICAÇÕES ORIGINAIS DAS ESPÉCIES}

TAXIDERMIZADAS", consta como “criticamente em perigo" Informação também encontrada em WIKIAVES ${ }^{12}$ :

O Mergus octosetaceus só sobrevive em ecossistemas ambientalmente equilibrados, em especial aqueles em que há cursos d'água limpos e transparentes. Por causa dessa peculiaridade - e dada a delicada situação dos recursos hídricos do planeta no século 21 -, não é de espantar que essa ave aquática seja classificada como "criticamente em perigo" nas listas das espécies consideradas em sério risco de extinção.

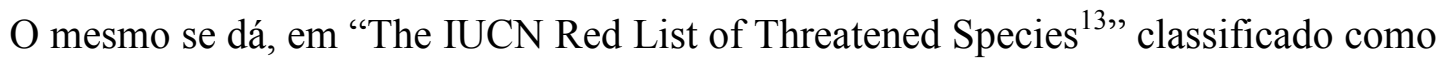
"Criticamente em risco", conforme mostra a imagem abaixo:

\footnotetext{
${ }^{11}$ ICMBIO Instituto Chico Mendes de Conservação da Biodiversidade. Ministério do Meio Ambiente. Disponível em: $\quad<$ http://www.icmbio.gov.br/portal/images/stories/comunicacao/publicacoes/publicacoesdiversas/dcom sumario executivo_livro_vermelho ed_2016.pdf $>$. Acesso em: 26 jun. 2017.

${ }^{12}$ WIKIAVES. Disponível em: $<$ http://www.wikiaves.com.br/pato-mergulhao $>$. Acesso em: 21 jun. 2017.

${ }^{13}$ The IUCN Red List of Threatened Species. Disponível em: <http://www.iucnredlist.org/details/22680482/0>. Acesso em: 26 jun. 2017.
} 
Figura 4. Mergus octosetaceus - STATUS DE “CRITICAMENTE EM RISCO” - 2016.

\begin{tabular}{|c|c|c|c|c|c|}
\hline VIEVV IVIAT & \multicolumn{5}{|l|}{ Taxonomy [top] } \\
\hline \multirow{7}{*}{ TAKE ACTION NOW } & Kingdom & Phylum & Class & Order & Family \\
\hline & Animalia & Chordata & Aves & Anseriformes & Anatidae \\
\hline & Scientific Name: & \multicolumn{4}{|c|}{ Mergus octosetaceus } \\
\hline & Species Authority: & \multicolumn{4}{|c|}{ Vieillot, 1817} \\
\hline & \multicolumn{5}{|c|}{$\begin{array}{l}\text { Common Name(s): } \\
\text { English - Brazilian Merganser }\end{array}$} \\
\hline & $\begin{array}{l}\text { Taxonomic } \\
\text { Source(s): }\end{array}$ & \multicolumn{4}{|c|}{$\begin{array}{l}\text { del Hoyo, J., Collar, N.J., Christie, D.A., Elliott, A. and Fishpool, L.D.C. 2014. HBW and } \\
\text { BirdLife International Illustrated Checklist of the Birds of the World. Volume 1: Non- } \\
\text { passerines. Lynx Edicions BirdLife International, Barcelona, Spain and Cambridge, UK. }\end{array}$} \\
\hline & $\begin{array}{l}\text { Identification } \\
\text { information: }\end{array}$ & \multicolumn{4}{|c|}{$\begin{array}{l}49-56 \mathrm{~cm} \text {. Dark, slender duck with long crest. Dark hood with petroleum-green sheen. } \\
\text { Pale grey breast finely vermiculated dark, paler towards whitish belly. Dark grey } \\
\text { upperparts. White wing speculum. Long, dark saw-bill. Pinky-lilac legs. Long, bushy } \\
\text { hindcrest usually worn and shorter in females. Similar spp. Neotropic Cormorant } \\
\text { Phalacrocorax brasilianus is larger and darker with hooked bill. Voice Harsh and dry } \\
\text { jrrec contact call. Also louder nasal juac calls. Hints Hides under overhanging } \\
\text { vegetation. }\end{array}$} \\
\hline
\end{tabular}

\section{Assessment Information [top]}

\begin{tabular}{|c|c|}
\hline $\begin{array}{l}\text { Red List Category \& } \\
\text { Criteria: }\end{array}$ & Critically Endangered C2a(i) ver 3.1 \\
\hline Year Published: & 2016 \\
\hline Date Assessed: & $2016-10-01$ \\
\hline Assessor(s): & BirdLife International \\
\hline Reviewer(s): & Butchart, S. \& Symes, A. \\
\hline Contributor(s): & $\begin{array}{l}\text { Bosso, A., Chebez, J., Clay, R., Gil, G., Silveira, L., Yamashita, C., Lamas, I., Lins, L., } \\
\text { Cockle, K., Disconzi, G. \& Diniz, M. }\end{array}$ \\
\hline Facilitator/Compiler(s): & $\begin{array}{l}\text { Benstead, P., Bird, J., Butchart, S., Calvert, R., Capper, D., Clay, R., Mazar Barnett, J., } \\
\text { Pilgrim, J., Sharpe, C J, Symes, A., Taylor, J., Khwaja, N. \& Ashpole, J }\end{array}$ \\
\hline \multicolumn{2}{|c|}{$\begin{array}{l}\text { Justification: } \\
\text { Recent records from Brazil indicate that this species's status may be marginally better than previously thought. } \\
\text { Nevertheless, the remaining known population is still extremely small and fragmented, and the perturbation, } \\
\text { damming and pollution of rivers are likely to be causing continuing declines. For these reasons, it is listed as } \\
\text { Critically Endangered. Further information on the population size (in particular whether it exceeds } 250 \text { mature } \\
\text { individuals) and on the subpopulation structure may result in its downlisting to Endangered in the future. }\end{array}$} \\
\hline
\end{tabular}

Fonte: The IUCN Red List of Threatened Species.

A Lista Vermelha de Espécies Ameaçadas da

União Internacional Para Conservação da Natureza e dos Recursos Naturais (IUCN) foi Criada em 1964 e tem como objetivo informar a sociedade e pesquisadores do planeta a respeito da conservação dos seres vivos. Ela apresenta 
informações relevantes a respeito da fauna e flora do planeta, mas não apresenta dados a respeito de micro-organismos 14 .

Ainda sobre esta mesma "Lista Vermelha de Espécies Ameaçadas da IUCN, criticamente em perigo (em inglês, Critically Endangered - CR): A espécie classificada como "criticamente ameaçada" corre um risco extremamente alto de ser extinta da natureza"15.

O pato mergulhão é uma ave rara que:

Vivia na Argentina, Paraguai e Brasil, mas hoje, os últimos 250 indivíduos de sua espécie estão apenas no Brasil. Cerca de metade habita a Serra da Canastra (MG) e outros poucos animais a Chapada dos Veadeiros (GO) e o Jalapão (TO). [...] foi encurralado pela falta de habitat adequado. [...] tem bico negro longo, estreito e serrilhado nas bordas. Cabeça e pescoço escuros, com reflexos verde-metalizados [...,]. O pato-mergulhão é extremamente sensível à degradação e perda de seu ambiente natural. Por isso, é considerado um bom indicador da qualidade dos ambientes aquáticos ${ }^{16}$.

Outro espécime, em extinção, do acervo dos animais taxidermizados da EE "Culto à Ciência", o Bugio Rugio, apresentado abaixo, também foi motivo de busca, consternação e alerta, sobretudo, diante da beleza e atuação na natureza deste e dos demais animais selecionados para este pequeno estudo.

\footnotetext{
${ }^{14}$ Mundo Educação. Lista Vermelha de Espécies Ameaçadas da IUCN. Disponível em: $<$ http://mundoeducacao.bol.uol.com.br/curiosidades/lista-vermelha-especies-ameacadas-iucn.htm $>$. Acesso em: 21 jun. 2017.

${ }^{15}$ Mundo Educação. Lista Vermelha de Espécies Ameaçadas da IUCN. Disponível em: $<$ http://mundoeducacao.bol.uol.com.br/curiosidades/lista-vermelha-especies-ameacadas-iucn.htm>. Acesso em: 21 jun. 2017.

${ }^{16}$ ANDA. Disponível em: <https://www.anda.jor.br/2013 jun. pato-mergulhao-e-uma-das-aves-mais-ameacadasdo-mundo/>. Acesso em: 04 jul. 2017.
} 


\section{BUGIO RUIVO (nome popular)}

Figura 5. Bugio.

Classificação Cientifica ${ }^{17}$ Reino: Animalia Filo: Chordata

Classe: Mammalia Ordem: Primates Família: Cebidae Nome Científico: Alouatta fusca Estado de Conservação: Ameaçada

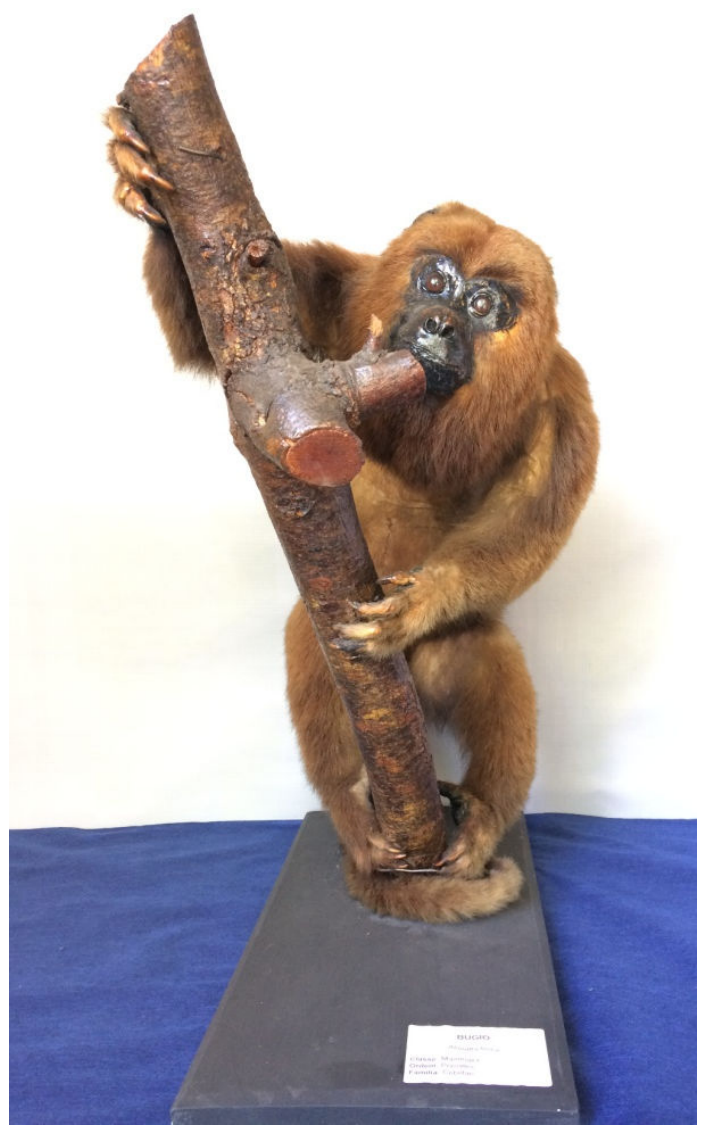

Fonte: Arquivo Histórico Documental da EE"Culto à Ciência" Campinas/SP Foto: Tamiris Pinheiro de Souza - PIBIC-EM/FAEPEX/UNICAMP.

Conforme a pasta "IDENTIFICAÇÕES ORIGINAIS DAS ESPÉCIES TAXIDERMIZADAS”, esta espécie encontra-se ameaçada de extinção. De acordo com CHIARELLO (1992), embora "alouatta fusca" seja o nome mais utilizado na literatura, o nome correto deve ser “A. guariba, Humboldt, 1812”. Com essa nomenclatura encontramos algumas referencias a este animal em várias fontes:

A "IUCN Red List of Threatened Species" ${ }^{18 "}$ o classifica como "Menor preocupação". Conforme comprova a imagem a seguir.

\footnotetext{
${ }^{17}$ Dados de acordo com pasta "IDENTIFICAÇÕES ORIGINAIS DAS ESPÉCIES TAXIDERMIZADAS".

${ }^{18}$ The IUCN Red List of Threatened Species. Disponível em: $<$ http://www.iucnredlist.org/details/39916/0 $>$.
} Acesso em: 26 jun. 2017. 
Figura 6. Alouatta guariba (sinônimo Alouatta fusca) - STATUS DE “MENOR PREOCUPAÇÃO” - 2008.

\begin{tabular}{|c|c|}
\hline Scientific Name: & Alovara guariba \\
\hline Species Authority: & (Humbolet 1812) \\
\hline $\begin{array}{l}\text { Infraspecific Taxa } \\
\text { Assessed: }\end{array}$ & 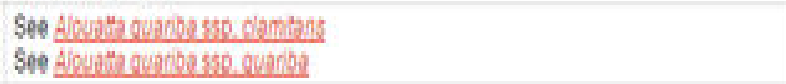 \\
\hline \multicolumn{2}{|c|}{$\begin{array}{l}\text { Common Name(s): } \\
\text { English - Brown Howler, Brown Howler Moniey, Brown Howling Moniey, Southem Brown Howling } \\
\text { Monigy } \\
\text { French - Hurleur Brun } \\
\text { Spanish - Barbaso, Bugo, Guariba }\end{array}$} \\
\hline Synonym(s): & Alovata fusca (E. Geotroy, 1812) \\
\hline Taxonomic Notes: & 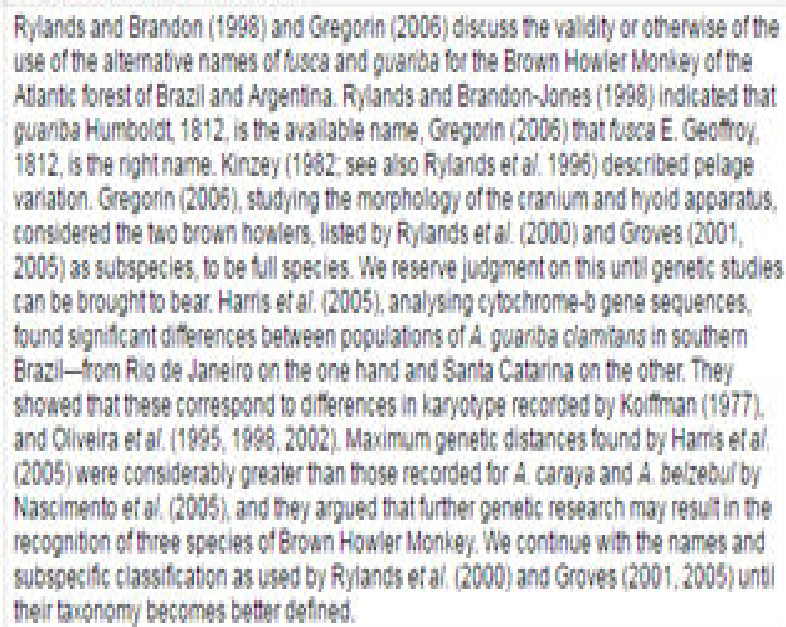 \\
\hline
\end{tabular}

Assessment information [loo]

\begin{tabular}{|c|c|}
\hline $\begin{array}{l}\text { Red List Category } \\
8 \text { Criteria: }\end{array}$ & Least Concern yer 31. \\
\hline Year Published: & 2008 \\
\hline Date Assessed: & $2008-06 \cdot 30$ \\
\hline Assessor(s)i & Mendes, S.L. Ry/ands AB, Keruit, M.C.M \& de Olveira, M.M \\
\hline Reviewer(s): & Mrtermelet, RA 8 Rylands, A. B. (Primate Red List Authonit) \\
\hline \multicolumn{2}{|c|}{ 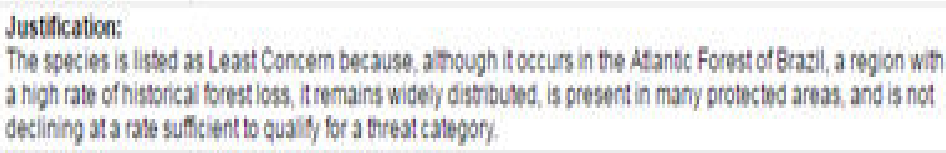 } \\
\hline $\begin{array}{l}\text { Previously } \\
\text { published Red List } \\
\text { assessments: }\end{array}$ & 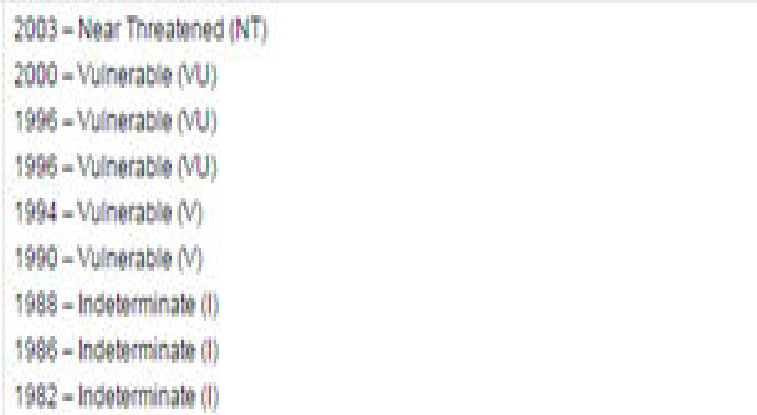 \\
\hline
\end{tabular}

Fonte: The IUCN Red List of Threatened Species. 
Por sua vez, o "Livro Vermelho da Fauna Brasileira Ameaçada de Extinção ${ }^{19 "}$ (p.45), classifica a espécie como "Ameaçada" em conformidade com a imagem abaixo.

Figura 7. Alouatta guariba guariba (Humboldt, 1812) - (sinônimo Alouatta fusca) - STATUS DE "AMEAÇA" 2007.

Livro Vermelho da Fauna Brasileira Ameaçada de Extinção

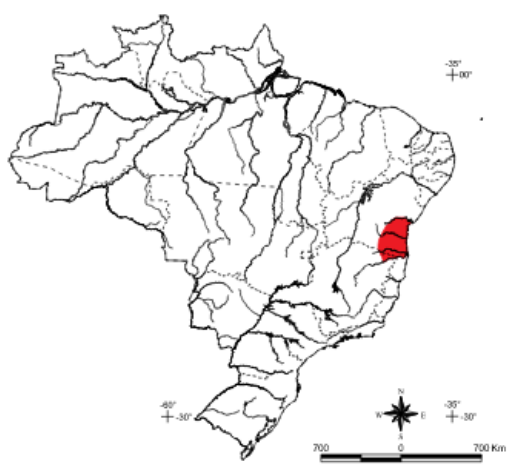

Alouatta guariba guariba (Humboldt, 1812)

NOME POPULAR: Bugio-marrom-do-norte; Guariba-marrom-do-norte

SINONIMIAS: Alouatta fusca fusca

FILO: Chordata

CLASSE: Mammalia

ORDEM: Primates

FAMÍLIA: Atelidae

STATUS DE AMEAÇA

Brasil (MMA, IN 03/03): Ameaçada

Estados Brasileiros: não consta

Anexos da CITES: Anexo II

CATEGORIAS RECOMENDADAS

Mundial (IUCN, 2007): CR

Brasil (Biodiversitas, 2002): CR - B2ab(i, ii, iii); C2a(i); D

Fonte: Livro Vermelho da Fauna Brasileira Ameaçada de Extinção.

\footnotetext{
${ }^{19}$ ICMBIO Instituto Chico Mendes de Conservação da Biodiversidade. Ministério do Meio Ambiente. Disponível em: $\quad<$ http://www.icmbio.gov.br/portal/images/stories/biodiversidade/fauna-brasileira/livrovermelho/volumeII/Mamiferos.pdf $>$. Acesso em: 26 jun. 2017.
} 
Livre na natureza, assim se locomove e se alimenta o bugio-ruivo. Cena real que pode estar fadada a deixar de existir, muito em breve...

Figura 8. Macho adulto de Alouatta fusca se alimentando de folhas jovens de Celtis iguanae (também chamada de Juá-mirim).

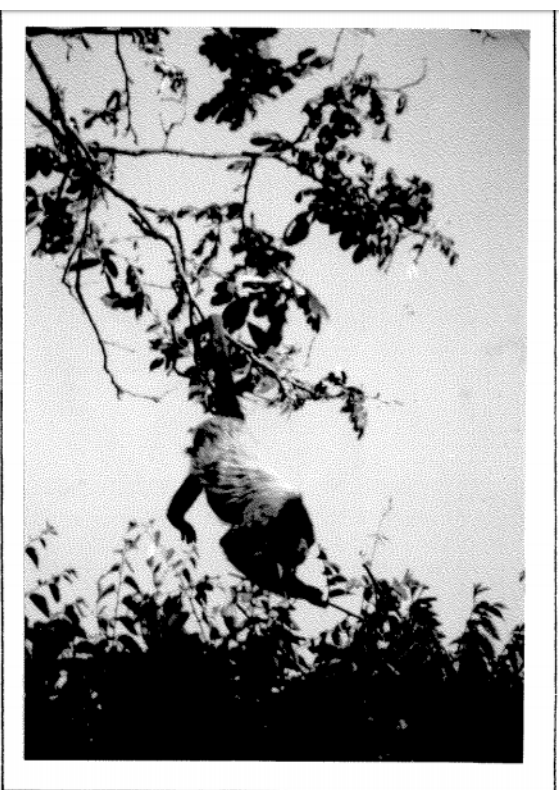

Fonte: Chiarello (1992, p. 2).

Os bugios,

[...] ocorrem principalmente no Brasil, Argentina e México, em florestas tropicais úmidas, e até mesmo em savanas [...] possuem hábitos herbívoros, corpo forte e cauda longa; com vasta pelagem que varia entre as colorações preta, marrom e vermelha [...]. Algumas espécies deste gênero, como a Alouatta caraya e Alouatta guariba clamitans, estão ameaçadas de extinção, principalmente pela perda de hábitats e caça indiscriminada, em virtude do uso de sua carne e pele por alguns grupos humanos. ${ }^{20}$

Manifestações sonoras - sua característica mais importante:

Seu rugido (um ronco forte), "é interrompido e recomeçado várias vezes durante até 30 minutos. Costuma ser emitido quando são observados outros grupos se aproximando ou com a invasão do território por outro indivíduo; sinal de perigo; marcação de território"21.

\footnotetext{
${ }^{20}$ Mundo Educação - Bugio (Gênero Alouatta). Disponível em: $<$ http://mundoeducacao.bol.uol.com.br/biologia/bugio.htm>. Acesso em: 04 jul. 2017.

${ }^{21}$ Embrapa - $\quad$ Monitoramento por Satélite. Disponível em: < http://www.faunacps.cnpm.embrapa.br/mamifero/bugio.html $>$. Acesso em: 04 jul. 2017.
} 


\section{TRISTE PARADOXO}

Diante destes elementos apresentados e representados, permanece o questionamento dos participantes de um projeto de preservação da cultura material escolar, sobre os exemplares de animais de nossa fauna que foram abatidos para compor um acervo museológico para que os escolares das décadas finais do século XIX e décadas do século XX pudessem conhecer e estudar as espécies apresentadas. Ao grupo do projeto, em especial, aos pesquisadores do Ensino Médio, permaneceu a indagação de quantos outros exemplares destes animais também foram abatidos e taxidermizados e se encontram em outras instituições que, tal como ocorre na EE "Culto à Ciência", buscam preservá-los, anunciando-os como últimos exemplares de espécies com status "Criticamente em Perigo" e "Ameaçado de Extinção".

Afugentando os possíveis anacronismos, visualizamos tal situação como um triste paradoxo.

Permanece, entretanto, o desafio à escola em conscientizar e, a nós e às novas gerações, o desafio em reivindicar a proteção de nossas espécies ameaçadas.

\section{REFERÊNCIAS}

ARQUIVO HISTÓRICO DOCUMENTAL, EE "Culto à Ciência". Pasta "Identificações Originais das Espécies Taxidermizadas”. Campinas/SP. S/d.

CHIARELLO, Adriano Garcia. Dieta, padrão de atividades e área de vida de um grupo de bugios (Alouatta fusca) na reserva de Santa Genebra, Campinas, SP. 1992. 93f. Dissertação (mestrado) - Universidade Estadual de Campinas, Instituto de Biologia, Campinas, SP.

FABICHAK, Irineu. ABC da taxidermia: (arte de empalhar animais). São Paulo, SP: Cupolo, 1969. 51 p., il.

INSTITUTO DE BIOLOGIA/Unicamp. Introdução taxidermia. Disponível em: $<$ http://www.ib.unicamp.br/dep_biologia_animal/taxidermia $>$. Acesso em: 21 jun. 2017.

LIVRO vermelho dos mamíferos brasileiros ameaçados de extinção. Coautoria de Gustavo A. B. da Fonseca. Belo Horizonte, MG: Fundação Biodiversitas, 1994. xx, 459p., il. Inclui bibliografia. (Broch).

MONTENEGRO, Mônica Mafra Valença. Ecologia de Cebus flavius (Schreber, 1774) em remanescentes de Mata Atlântica no estado da Paraíba. Tese de Doutorado. Piracicaba, 2011. Disponível em <http://www.teses.usp.br/teses/disponiveis/91/91131/tde-20122011143229/pt-br.php >. Acesso em: 21 jun. 2017. 
MUSEU de Ciências Morfológicas - UFRN. Disponível em $<$ http://www.clientesinterativa.com.br/museu/noticia-interna.php?idNot $=25548>$. Acesso em: 21 jun. 2017.

ORRELL, T. (custodian). ITIS Global: The Integrated Taxonomic Information System (version Jun 2017). In: ROSKOV, Y. et al. Species 2000 \& ITIS Catalogue of Life. 29th May 2017. Digital resource at $<$ www.catalogueoflife.org/col $>$. Species 2000: Naturalis, Leiden, the Netherlands.

VILLAVERDE, Alejandro. El art de disecar: taxidermia. 4. ed. Barcelona: Sintes, 1968. 268p., il. (Biblioteca de animales utiles). (Broch). 